\title{
Wrinkles in the rare biosphere: Pyrosequencing errors can lead to artificial inflation of diversity estimates
}

Victor Kunin ${ }^{1}$, Anna Engelbrektson ${ }^{1}$, Howard Ochman ${ }^{2}$, and Philip Hugenholtz ${ }^{1 \Uparrow}$

${ }^{1}$ Microbial Ecology Program, DOE Joint Genome Institute, Walnut Creek,

$1094598 \mathrm{CA}, \mathrm{USA},{ }^{2}$ Department of Chemistry and Biochemistry, University of Arizona, Tucson, AZ, USA.

『Corresponding author: fax 925-296-5720•email: phugenholtz@1bl.gov

Email addresses:

15 Victor Kunin: vkunin@1bl.gov

Anna Engelbrektson: AEngelbrektson@lbl.gov

Howard Ochman: hochman@email.arizona.edu

Philip Hugenholtz: PHugenholtz@lbl.gov 


\section{SUMMARY}

Massively parallel pyrosequencing of the small subunit (16S) ribosomal RNA gene has revealed that the extent of rare microbial populations in several environments, the "rare biosphere", is orders of magnitude higher than previously thought. One important caveat with this method is that sequencing error could artificially inflate diversity estimates.

25 Although the per-base error of $16 \mathrm{~S}$ rDNA amplicon pyrosequencing has been shown to be as good as or lower than Sanger sequencing, no direct assessments of pyrosequencing errors on diversity estimates have been reported. Using only Escherichia coli MG1655 as a reference template, we find that $16 \mathrm{~S}$ rDNA diversity is grossly overestimated unless relatively stringent read quality filtering and low clustering thresholds are applied. In

30 particular, the common practice of removing reads with unresolved bases and anomalous read lengths is insufficient to ensure accurate estimates of microbial diversity. Furthermore, common and reproducible homopolymer length errors can result in relatively abundant spurious phylotypes further confounding data interpretation. We suggest that stringent quality-based trimming of $16 \mathrm{~S}$ pyrotags and clustering thresholds

35 no greater than $97 \%$ identity should be used to avoid overestimates of the rare biosphere.

\section{INTRODUCTION}

Pyrosequencing (Margulies et al., 2005) is one of the leading technologies supplanting Sanger sequencing for comparative genomics and metagenomics. One emerging

40 application is the pyrosequencing of $16 \mathrm{~S}$ rRNA genes ("16S pyrotags") to profile the phylogenetic diversity within microbial communities. The large number of reads produced in a single pyrosequencing run provides unprecedented sampling depth, leading to the conclusion that the rare biosphere, i.e. the tail of the species abundance distribution, is substantially larger and more diverse than previously appreciated (Sogin et 45 al., 2006).

One caveat, however, is that the intrinsic error rate of pyrosequencing could lead to overestimates of the number of rare phylotypes. Unlike genome sequencing projects in which sequencing errors can be corrected by assembly and sequencing depth, each read in a pyrotag analysis is interpreted as a unique identifier of a community member and 
50 therefore errors will potentially inflate diversity estimates. Sogin and coworkers, appreciating this risk, invested considerable effort to determine the error rates of first generation GS20 pyrosequencing using a mixture of 43 reference templates (Huse et al., 2007). They concluded that quality filtering based on the removal of reads with one or more unresolved bases (N's), errors in the barcode or primer sequence, and/or atypically

55 short or long reads is sufficient to ensure per-base error rates lower than conventional Sanger sequencing while retaining $>90 \%$ of the reads. Ideally, the number of operational taxonomic units (OTUs) from their analysis should have been 43, however they did not report OTU estimates of their synthetic community based on pre- or post-filtered pyrosequencing reads. Here we assess the effect of error rates in second generation FLX

60 pyrosequencing on diversity estimates using pyrotags PCR-amplified from two regions of the 16S rRNA amplicons of a well-characterized laboratory isolate of E. coli.

\section{Results}

Approximately 300-bp regions from the 5' and 3' ends of the 16S rRNA genes of E. coli

65 MG1655 were PCR-amplified using adaptor-modified standard primer sets (A-27F/B$342 \mathrm{R}$ and B-1114F/A-1392R) and pyrosequenced from the 27-forward or 1392-reverse primers, producing a total of 9,781 reads. Of these, 4,254 and 4,244 (87\% of the total reads) could be unambiguously assigned to the 5'-forward and 3'-reverse regions of the 16S rRNA molecule, respectively, based on the presence of error-free barcode and primer 70 sequences.

Read quality filtering. Reads were quality filtered by applying either the current practice of removing reads with unresolved bases and/or anomalous read length, or quality scorebased end-trimming at different stringencies (3\% to $0.1 \%$ per base error probabilities). After quality filtering and trimming to a uniform length of $244 \mathrm{bp}$ to enable comparisons

75 across samples and regions, the resulting reads were compared to the 16S rRNA sequences from the E. coli MG1655 genome to determine error rates. The extent of improvement and data loss after applying such quality filtering and length trimming is presented in Figure 1. The 5'-forward region had, on average, 15\% more reads with one or more errors than did the 3 '-reverse region at each quality-filtering treatment (Table 1).

80 This difference is due to the higher number of homopolymers in the 5'-forward region 
relative to the 3' region (62 vs. 50), because homopolymer miscounts are the major source of errors in pyrosequence data (Margulies et al., 2005; Huse et al., 2007).

The lower quality of data from the 5'-forward region resulted in $\sim 15 \%$ fewer usable reads than from the 3'-reverse region. The now standard practice of removing 85 reads with undetermined bases (i.e., N's) resulted in only a marginal improvement ( 1\%) in errorless reads. In contrast, we found that trimming based on quality scores had a more pronounced effect on error rate when relatively stringent per-base error probabilities were applied $(\leq 0.2 \%$ producing $>4 \%$ improvement in errorless reads; Table 1$)$. The number of usable reads decreased sharply when the most stringent $(0.1 \%)$ error probability was

90 applied, indicating that the benefits of increasing the stringency of quality filtering stringency were not offset by data loss beyond $0.2 \%$ error probability for this dataset. Clustering evaluation. Reads were aligned and clustered at various identity thresholds ranging from $100 \%$ (unique sequences) down to $90 \%$ (sequences that differ by $10 \%$ are clustered into a single OTU) (Table 1, Fig. 1). Assuming no sequencing errors, the

95 theoretical number of clusters (OTUs) should correspond to the actual number of $16 \mathrm{~S}$ phylotypes in the sample; and in the case of E. coli MG1655, the number of unique OTUs should be five in the 5'-forward region and one in 3'-reverse region (Table 1).

Remarkably, unfiltered reads overestimate this diversity by two orders of magnitude, producing 643 and 385 unique OTUs from the 5'-forward and 3'-reverse regions,

100 respectively (Table 1, Fig. 1). Moreover, we note that increases in the size of the dataset will increase the observed number of OTUs (Fig. S1).

In ranking the abundance of OTUs in our samples, the majority of reads possess the exact sequence of the corresponding region in an $E$. coli 16S rRNA gene; however, rank-abundance distributions for both regions were flanked by a long tail of OTUs

105 containing one or more insertion and/or substitution errors relative to the E. coli reference sequences, and in the case of the 5'-forward region, two putative chimeric OTUs formed between different E. coli 16S operons (Fig. 2). A remarkable feature of the 5'-forward region distribution is that between the abundant error-free OTUs and the rare erroneous OTUs and singletons, there were several moderately abundant clusters, together 110 constituting $\sim 6 \%$ of the reads. These OTUs contain the same re-occurring homopolymer error; 6 instead of 5 guanines spanning E. coli positions 200 to 204 (Fig. 2). 
The primary effect of clustering at different levels of sequence identity was to recruit erroneous OTUs and singletons into larger clusters, thereby decreasing exponentially the number of OTUs as identity thresholds were relaxed (Fig. 1). But even

115 at the most relaxed threshold, there were two 5'-forward and one 3'-reverse OTUs that did not match E. coli. The closest matches ( $>98 \%$ identity) to these OTUs were members of the Saprospirales (Bacteroidetes), Bradyrhizobiales (Alphaproteobacteria) and Peptostreptococcaceae (Firmicutes). All other sequences clearly originated from E. coli and represent the overwhelming majority $(99.97 \%)$ of the sequence data.

120

\section{DISCUSSION}

Despite a rigorous analysis of error rates in 16S rRNA pyrosequences of known templates (Huse et al., 2007), there have been no reports of the effect of pyrosequencing errors on diversity estimates (number of inferred phylotypes), and therefore, no way to

125 gauge the accuracy of diversity reported in individual studies or to compare the observed variation of communities across studies. To resolve this issue, we chose to examine a single bacterial strain both to remove the complication of inter-species chimera formation (Huber et al., 2004) and to focus solely on the effect of pyrosequencing error on diversity estimates. Even with a fairly modest number of second generation 454 FLX reads from

130 two regions of the 16S rRNA genes of Escherichia coli MG1655 ( $\sim 4250$ reads per region), we find that sequencing errors inflate estimates of the actual diversity by two orders of magnitude when considering unique reads (Fig. 1).

This overestimation is consistent with a high percentage of reads with one or more errors; $\sim 15 \%$ and $\sim 30 \%$ of reads for the 3 '-reverse (V8) and 5 '-forward (V1\&2)

135 regions, respectively (Table 1) also detected in prior analysis of the V6 region in which $18 \%$ of reads had $\geq 1$ error (Huse et al., 2007). A large proportion of these artefacts is attributable to miscounted homopolymeric runs that occur in otherwise high quality regions of the read, and are therefore not removed by end-trimming based on quality scores (see below) or by culling reads with unresolved bases or anomalous lengths.

140 Moreover, some of these errors are highly reproducible and produce phantom OTUs with large numbers of reads (Fig. 2), indicating that not only will false phylotypes be detected, 
but that, in some cases, spurious phylotypes will be relatively abundant $(\geq 1 \%)$ at least in the case of $100 \%$ OTUs.

In practice, $100 \%$ sequence identity is rarely used as a threshold for defining

145 OTUs, but rather, reads are usually grouped at some lower level of sequence identity (often 97\% sequence identity (Stackebrandt and Goebel, 1994), which clusters sequences differing by as much as 3\% into a single OTU). This has the effect of absorbing much of the observed sequencing errors. We tested a range of clustering thresholds, and as expected, clustering greatly reduces the overestimation of diversity (Fig. 1). However, we

150 find that the current practice of removing reads with undetermined bases and/or anomalous read lengths is not adequate to ensure accurate diversity estimates at a $97 \%$ clustering threshold (Fig. 1). This occurs despite the comparable or lower per base error rates observed for 454 pyrosequencing when compared to conventional Sanger sequencing (Huse et al., 2007).

Recent improvements in error estimation of pyrosequence data (Brockman et al., 2008) allow the use of trimming programs, such as LUCY (Chou and Holmes, 2001), that are based on the per-nucleotide quality score. Only when the LUCY end-trimming stringency was increased to $\leq 0.2 \%$ per base error probability (equivalent to a phred quality score of $\geq 27$ ), combined with clustering at $\leq 97 \%$ identity, did the number of

160 OTUs approach the expected number of $E$. coli MG1655 rRNA operons. The slightly overestimated number of OTUs at these settings were, in fact, not sequencing artefacts, but most likely due to experimental contamination introduced during the PCR amplification, as seen previously with no-template PCR controls (Tanner et al., 1998). These contaminants represent only $0.03 \%$ of the reads obtained in the present study and

165 suggest that all PCR-based surveys that use broad-specificity primers will likely suffer from similar low-level background contamination, a point worth bearing in mind when interpreting rare biosphere data.

Based on our analyses, we propose the use of quality trimming to $0.2 \%$ error probability and a clustering threshold of $97 \%$ identity when applying 454 pyrosequencing 170 to community profiling. These parameters should substantially reduce artefactual inflation of diversity estimates due to pyrosequencing errors. Raising the trimming stringency from $0.2 \%$ to $0.1 \%$ error probability results in a sharp decrease in usable reads 
with little additional improvement in error reduction (Table 1). We note, however, that error rates are sequence specific (Fig. 1a vs. 1b) and that the spurious inflation of OTU 175 numbers will increase with the size of the dataset (Fig. S1). Therefore, the proposed parameters may be insufficient to prevent overestimates of diversity using very large pyrotag datasets from regions of the 16S rRNA gene with a high fraction of homopolymers. Overall, we anticipate that the use of high stringency quality-based trimming and clustering thresholds $\leq 97 \%$ will be the simplest, least computationally

180 intensive means to ensure that $16 \mathrm{~S}$ pyrotag analyses provide accurate, high sensitivity phylogenetic profiling of microbial communities.

\section{MATERIALS AND METHODS}

DNA Extraction. Escherichia coli MG1655 was grown overnight at $37^{\circ} \mathrm{C}$ in $10 \mathrm{ml}$ of LB and harvested by centrifugation at 10,000xg for $5 \mathrm{~min}$. Cells were treated with

185 proteinase $\mathrm{K}(20 \mathrm{mg} / \mathrm{ml})$ and lysozyme $(5 \mathrm{mg} / \mathrm{ml})$, and DNA was isolated using a standard phenol-chloroform extraction, followed by ethanol precipitation.

PCR Amplicon Library Construction and Sequencing. One 5' and one 3' region of the 16S rRNA gene were targeted using the broad-specificity oligonucleotide primer pairs 27F/342R and 1114F/1392R (Stackebrandt and Goodfellow, 1991). Primer

190 sequence (small caps) were modified by addition of the Roche 454 A or B adaptor sequences (lower case) and a five nucleotide identifying barcode (bolded uppercase) to distinguish different amplicons in the same sequencing reaction, as follows: A-27F, 5'gec tcc ctc geg cca tca gAC GTC AGA GTT TGA TCM TGG CTC AG-3', B-342R, 5' 'gcc ttg cca gec cgc tca gCT GCT GCS YCC CGT AG-3', A-1392R, 5' -gcc tcc ctc geg cca tca gTG

195 CTG ACG GGC GGT GTG TRC-3' and B-1114F, 5' -gec ttg cea gec cge tca gGC AAC GAG CGC AAC CC-3'. $20 \mu \mathrm{L}$ PCR reactions were performed in triplicate for each primer pair, using 0.5 units Taq (GE Healthcare), $2 \mu \mathrm{L}$ of supplied 10X buffer, $0.4 \mu \mathrm{L}$ of $10 \mathrm{mM}$ dNTP mix (MBI Fermentas), $0.6 \mu \mathrm{L}$ of $10 \mathrm{mg} / \mathrm{mL}$ BSA (New England Biolabs), $0.2 \mu \mathrm{L}$ of each $10 \mu \mathrm{M}$ primer, and $10 \mathrm{ng}$ of $E$. coli genomic DNA per reaction. Thermocycling

200 proceeded as follows: $95^{\circ} \mathrm{C}$ for 3 mins followed by 30 cycles of $95^{\circ} \mathrm{C}$ for $30 \mathrm{sec}, 55^{\circ} \mathrm{C}$ for $45 \mathrm{sec}$, and $72^{\circ} \mathrm{C}$ for $90 \mathrm{sec}$ and final extension at $72^{\circ} \mathrm{C}$ for $10 \mathrm{~min}$. Upon completion, the three reactions for each primer pair were pooled, and amplicons were purified with the Qiagen MinElute PCR cleanup kit and quantified on a Qubit fluorometer (Invitrogen). 
Barcoded amplicons were mixed in equal proportions prior to emulsion PCR in

205 preparation for GS FLX pyrosequencing.

Informatic Analysis. Pyrosequencing flowgrams were converted to sequence reads using the standard software provided by 454 Life Sciences. Reads were either used directly (which served as the unfiltered control) or quality filtered in one of two ways: $(i)$ reads with any unresolved nucleotides (N's) were removed from the dataset, or (ii) reads were

210 end trimmed based on quality scores over a range of accuracy thresholds $(0.1$ to $3 \%$ per base error probabilities) using LUCY (Chou and Holmes, 2001). This resulted in eight quality filtered datasets (Table 1).

To compare sequences across samples, all reads in each of the datasets were truncated from their 3'end to $244 \mathrm{bp}$, and reads less than $244 \mathrm{bp}$ were discarded. In the

215 same step, barcodes and primer sequences were trimmed from the 5 ' end, and any read with a sequence error in its barcode and/or primer was removed. This resulted in 5'forward reads spanning positions 28 to 246 (E. coli numbering), which encompasses variable regions 1 and 2, and the 3' reads spanning positions 1168 to 1391 , which encompasses variable region 8 of the 16S rRNA molecule. From the remaining uniform

220 length sequences, all redundant sequences were removed yielding a dereplicated dataset containing only unique phylotypes (termed the 100\% OTUs in subsequent steps).

Unique truncated reads were aligned using a modified Needleman-Wunsch algorithm (Needleman and Wunsch, 1970) and clustered along a range of identity thresholds (90, 95, 97, 98 and 99\%) using MCL, executed with default parameters (Van

225 Dongen, 2000). Sequencing errors in each of the unique reads were determined by BLAST alignment (Altschul et al., 1997) to the known 16S rRNA gene sequences of $E$. coli MG1655, assuming that any mismatches derived from the most similar of the seven E. coli operons. For the 5'-forward region considered, there are five unique $16 \mathrm{~S}$ rRNA sequences in $E$. coli, and for the 3'-reverse region considered, all E. coli $16 \mathrm{~S}$ sequences

230 are identical. A subset of reads was manually inspected in ARB (Ludwig et al., 2004) to confirm the specific type and location of the BLAST-determined errors and to identify putative chimeras. 


\section{ACKNOWLEDGMENTS}

235 We thank Suzan Yilmaz for extracting E. coli DNA and Alex Copeland for discussions on quality-score based filtering. This work was performed under the auspices of the US Department of Energy's Office of Science, Biological and Environmental Research Program, and by the University of California, Lawrence Berkeley National Laboratory under contract No. DE-AC02-05CH11231, Lawrence Livermore National Laboratory

240 under Contract No. DE-AC52-07NA27344, and Los Alamos National Laboratory under contract No. DE-AC02-06NA25396. VK was supported in part by NSF grant OPP0632359.

\section{REFERENCES}

245 Altschul, S.F., Madden, T.L., Schaffer, A.A., Zhang, J., Zhang, Z., Miller, W., and Lipman, D.J. (1997) Gapped BLAST and PSI-BLAST: a new generation of protein database search programs. Nucleic Acids Res 25: 3389-3402.

Brockman, W., Alvarez, P., Young, S., Garber, M., Giannoukos, G., Lee, W.L. et al. (2008) Quality scores and SNP detection in sequencing-by-synthesis systems. Genome

250 Res 18: 763-770.

Chou, H.H., and Holmes, M.H. (2001) DNA sequence quality trimming and vector removal. Bioinformatics 17: 1093-1104.

Huber, T., Faulkner, G., and Hugenholtz, P. (2004) Bellerophon: a program to detect chimeric sequences in multiple sequence alignments. Bioinformatics 20: 2317-2319.

255 Huse, S.M., Huber, J.A., Morrison, H.G., Sogin, M.L., and Welch, D.M. (2007) Accuracy and quality of massively parallel DNA pyrosequencing. Genome Biol 8: R143. Ludwig, W., Strunk, O., Westram, R., Richter, L., Meier, H., Yadhukumar et al. (2004) ARB: a software environment for sequence data. Nucleic Acids Res 32: 1363-1371. Margulies, M., Egholm, M., Altman, W.E., Attiya, S., Bader, J.S., Bemben, L.A. et al.

260 (2005) Genome sequencing in microfabricated high-density picolitre reactors. Nature 437: 376-380.

Needleman, S.B., and Wunsch, C.D. (1970) A general method applicable to the search for similarities in the amino acid sequence of two proteins. J Mol Biol 48: 443-453. Sogin, M.L., Morrison, H.G., Huber, J.A., Mark Welch, D., Huse, S.M., Neal, P.R. et al. 265 (2006) Microbial diversity in the deep sea and the underexplored "rare biosphere". Proc Natl Acad Sci U S A 103: 12115-12120.

Stackebrandt, E., and Goodfellow, M. (1991) Nucleic acid techniques in bacterial systematics. Chichester; New York: Wiley.

Stackebrandt, E., and Goebel, B.M. (1994) Taxonomic Note: A Place for DNA-DNA

270 Reassociation and 16S rRNA Sequence Analysis in the Present Species Definition in Bacteriology. Int J Syst Bacteriol 44: 846-849. 
Tanner, M.A., Goebel, B.M., Dojka, M.A., and Pace, N.R. (1998) Specific ribosomal DNA sequences from diverse environmental settings correlate with experimental contaminants. Appl Environ Microbiol 64: 3110-3113.

275 Van Dongen, S. (2000) Graph Clustering by Flow Simulation. In: University of Utrecht. 


\section{FIGURES AND LEGENDS}

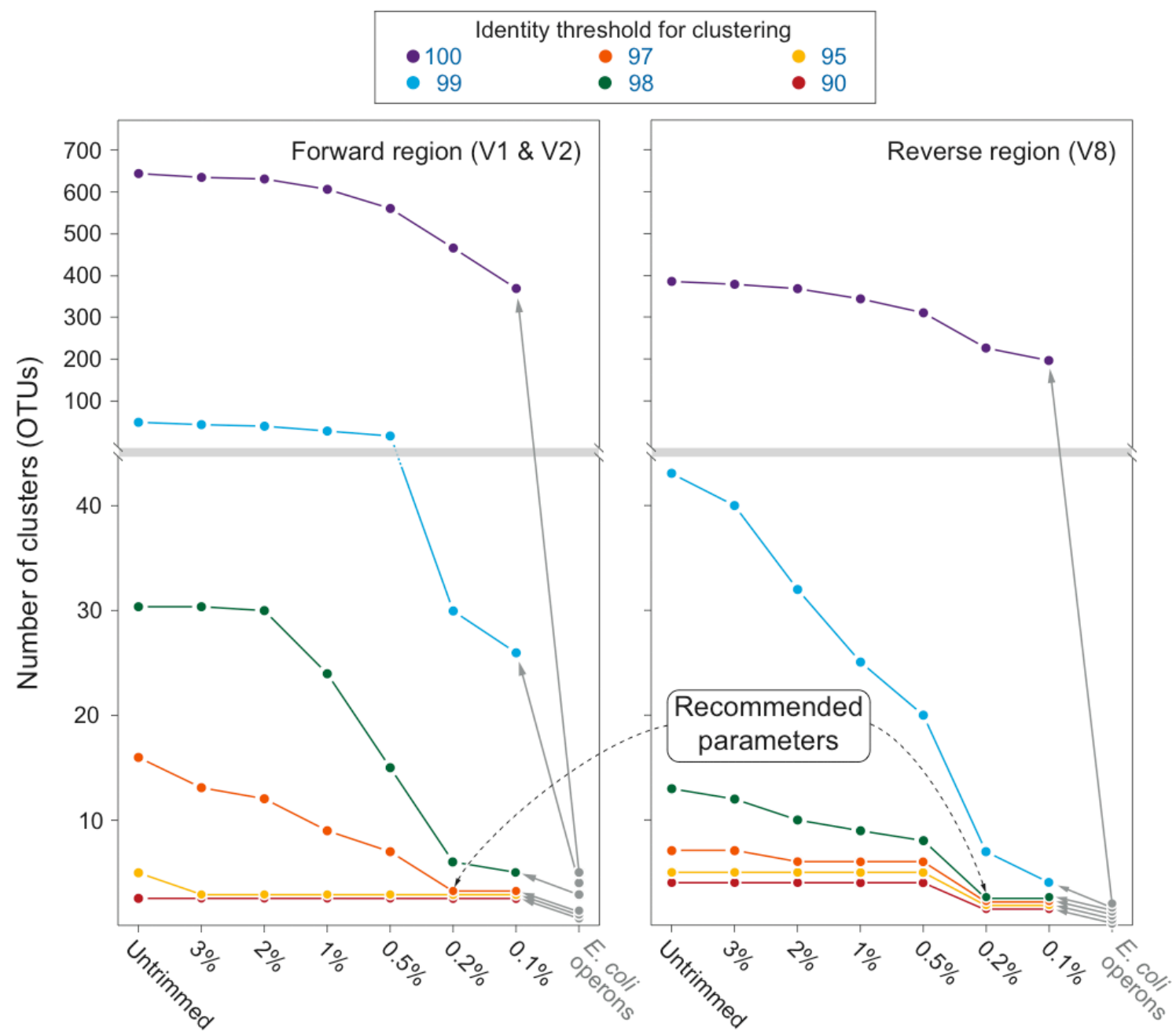

Quality trimming - error probability per nucleotide

Fig. 1. Graphical representation of effect of quality filtering and clustering on diversity estimates of an E. coli "community" using pyrotags from a 5'-forward (1A) and 3'reverse (1B) region of the $16 \mathrm{~S}$ rRNA molecule. 


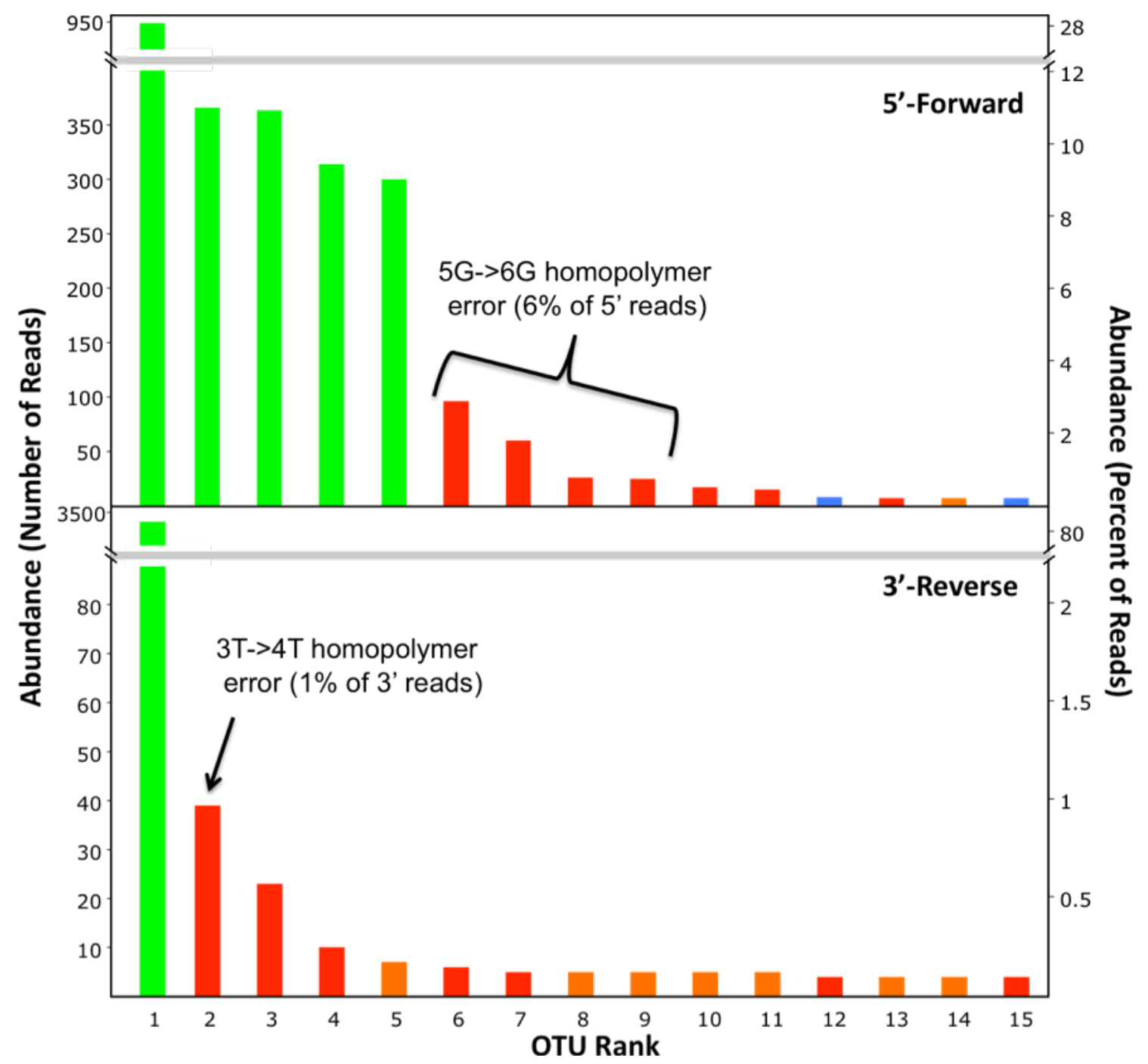

285 Fig. 2. Rank abundance distribution and error types of the top 15 unique phylotypes (100\% OTUs) from unfiltered 5'-forward and 3'-reverse 16S pyrotags. Colors denote errorless (green) reads, chimeras (blue) and reads with homopolymer length (red) or substitution (orange) errors. 
Table 1. Effect of quality filtering and clustering on diversity estimates (OTU number), error rate and data loss of pyrotags amplified from two regions of $E$. coli MG1655 16S rRNA genes. Diversity estimates should be considered relative to the theoretical number of OTUs from E. coli.

\begin{tabular}{|c|c|c|c|c|c|c|c|c|c|}
\hline \multirow{2}{*}{\multicolumn{2}{|c|}{ Read filtering }} & \multicolumn{6}{|c|}{ Number of OTUs at \% identity thresholds } & \multirow{2}{*}{$\begin{array}{c}\text { \% errorless } \\
\text { reads }\end{array}$} & \multirow{2}{*}{$\begin{array}{c}\% \text { reads } \\
\text { used }\end{array}$} \\
\hline & & 100 & 99 & 98 & 97 & 95 & 90 & & \\
\hline \multicolumn{10}{|c|}{$5^{\prime}$ forward $(\mathrm{V} 1 \& 2)$} \\
\hline \multicolumn{2}{|c|}{ theoretical number } & 5 & 4 & 3 & 1 & 1 & 1 & & \\
\hline \multicolumn{2}{|c|}{ no quality filtering } & 643 & 95 & 31 & 16 & 5 & 3 & 68.7 & 77.9 \\
\hline \multicolumn{2}{|c|}{ reads with Ns removed } & 600 & 85 & 29 & 14 & 4 & 3 & 69.8 & 76.7 \\
\hline \multirow{6}{*}{$\begin{array}{l}\text { quality score- } \\
\text { based filtering } \\
\text { (\% per base } \\
\text { error } \\
\text { probability) }\end{array}$} & 3 & 638 & 92 & 31 & 13 & 3 & 3 & 68.9 & 77.7 \\
\hline & 2 & 632 & 90 & 30 & 14 & 3 & 3 & 69.0 & 77.6 \\
\hline & 1 & 609 & 79 & 24 & 9 & 3 & 3 & 69.1 & 77.3 \\
\hline & 0.5 & 562 & 66 & 15 & 7 & 3 & 3 & 70.7 & 75.3 \\
\hline & 0.2 & 469 & 30 & 6 & 3 & 3 & 3 & 73.2 & 70.8 \\
\hline & 0.1 & 372 & 26 & 5 & 3 & 3 & 3 & 77.8 & 57.8 \\
\hline \multicolumn{10}{|c|}{ 3' reverse (V8) } \\
\hline \multicolumn{2}{|c|}{ theoretical number } & 1 & 1 & 1 & 1 & 1 & 1 & & \\
\hline \multicolumn{2}{|c|}{ no quality filtering } & 385 & 43 & 13 & 7 & 5 & 4 & 84.6 & 94.4 \\
\hline \multicolumn{2}{|c|}{ reads with Ns removed } & 361 & 40 & 12 & 6 & 4 & 3 & 85.3 & 93.6 \\
\hline \multirow{6}{*}{$\begin{array}{l}\text { quality score- } \\
\text { based filtering } \\
\text { (\% per base } \\
\text { error } \\
\text { probability) }\end{array}$} & 3 & 378 & 40 & 12 & 7 & 5 & 4 & 84.8 & 94.2 \\
\hline & 2 & 368 & 32 & 10 & 6 & 5 & 4 & 85.1 & 93.8 \\
\hline & 1 & 342 & 25 & 9 & 6 & 5 & 4 & 85.3 & 93.3 \\
\hline & 0.5 & 310 & 20 & 8 & 6 & 5 & 4 & 87.5 & 89.5 \\
\hline & 0.2 & 236 & 7 & 2 & 2 & 2 & 2 & 89.6 & 82.1 \\
\hline & 0.1 & 196 & 4 & 2 & 2 & 2 & 2 & 90.7 & 70.6 \\
\hline
\end{tabular}


Wrinkles in the rare biosphere

Kunin et al.

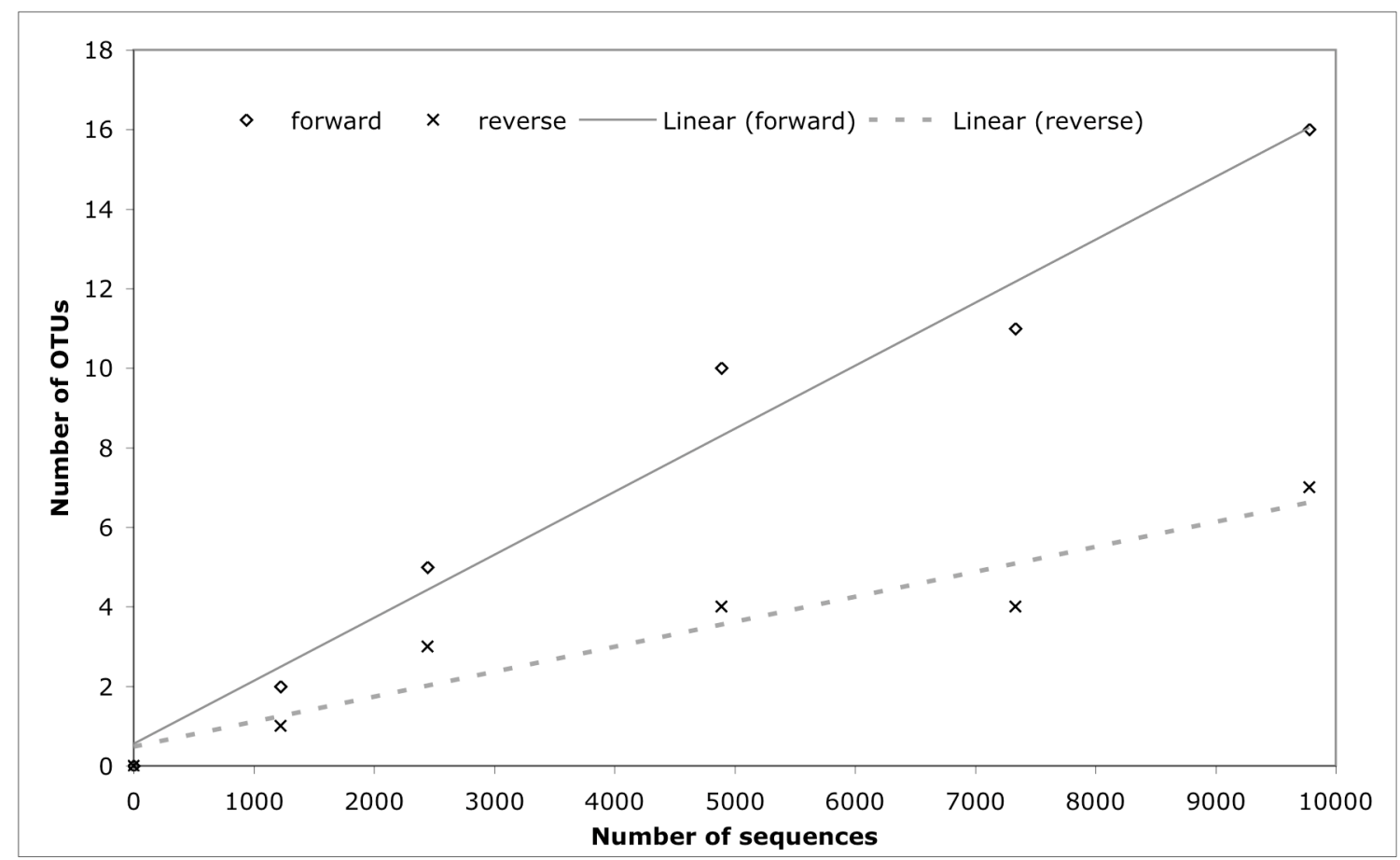

Fig. S1. Effect of pyrotag sample size on OTU number estimates from the 5'-forward and 3'-reverse regions of $E$. coli $16 \mathrm{~S}$ rRNA genes. 